\title{
Sommerschnitt von Hortensien für eine Blütenbildung spät im Jahr
}

\author{
Dominik Heukemes
}

\begin{abstract}
Hydrangeas are popular garden plants. They can be cut back differently, which influences their growth, habitus and flowers. By cutting in summer hydrangeas can bloom again in autumn. When choosing suitable nectar producing cultivars with fertile flowers such as Hydrangea paniculata 'Levana', 'Great Star' and 'Vanille Fraise', insects are offered a welcome food plant even late in the year.
\end{abstract}

\section{Zusammenfassung}

Hortensien sind beliebte Gartenpflanzen. Sie können unterschiedlich stark zurückgeschnitten werden, was ihren Wuchs sowie die Blütenbildung beeinflusst. Durch einen Rückschnitt im Sommer können Hortensien noch einmal im Herbst blühen. Bei der Wahl geeigneter nektarhaltiger Sorten mit fertilen Blüten wie Hydrangea paniculata 'Levana', 'Great Star' und 'Vanille Fraise' wird Insekten auch noch spät im Jahr eine willkommene Nahrungspflanze angeboten.

\section{Das Schneiden von Hortensien}

Immer wieder stellt sich Gartenfreundinnen und Gartenfreunden die Frage, wann und ob überhaupt Hortensien geschnitten werden sollten. Ein entsprechender Schnitt zur passenden Jahreszeit kann mitunter die Blühzeit verlängern und je nach Sorte Insekten ein zusätzliches Nektarangebot bieten.
Prinzipiell lassen sich alle Hortensien schneiden, man muss nur wissen wie. Die in Gärten häufig anzutreffende Rispen-Hortensie (Hydrangea paniculata) und deren Sorten sind sogar hervorragend für den Schnitt geeignet. Ein guter Zeitpunkt hierfür ist der ausgehende Winter oder der beginnende Frühling (je nach Witterungsverlauf

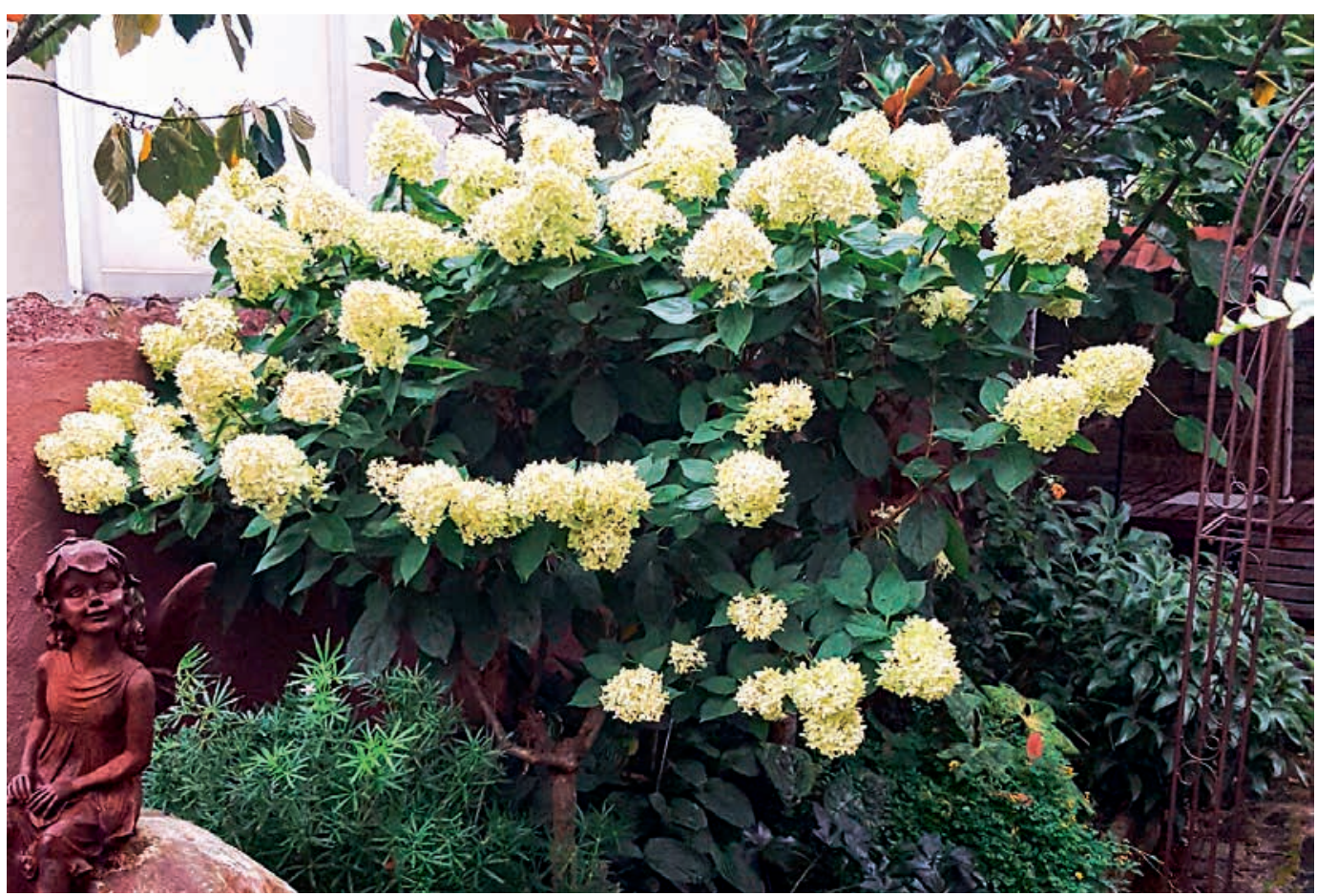

Abb. 1: An nicht zu sonnigen Standorten fühlen sich Hortensien wie Hydrangea paniculata 'Limelight' sehr wohl und bereichern den Garten. (Foto: D. Heukemes) 
etwa Mitte bis Ende März). Ein großer Vorteil der Rispenhortensie im Vergleich zur Bauern- oder Ballhortensie (Hydrangea macrophylla) besteht darin, dass sie auch nach einem kräftigen Rückschnitt blüht. Hortensien können verschieden stark zurückgeschnitten werden.

\section{Leichter Rückschnitt}

Es werden nur verblühte Blüten und tote Äste entfernt. Dies hat zur Folge, dass die Pflanze dann auch ihre tatsächliche Größe (je nach Sorte 2-3 m) erreichen kann. Der Nachteil eines solchen Schnittes ist, dass die Pflanzen sich relativ schnell verzweigen und dadurch die Blütenköpfe im Laufe der Zeit immer kleiner werden, dafür aber auch immer zahlreicher.

\section{Mittlerer Rückschnitt}

Dabei werden die Pflanzen etwa um die Hälfte der Gesamtlänge zurückgeschnitten. Dies bewirkt, dass die Pflanze danach stark austreibt und anschließend im Sommer sehr schöne große Blütenstände hervorbringt. Dieser Rückschnitt sorgt aber trotzdem für eine gute Stabilität der Pflanze, gerade was die Wind- und Regenlast der Pflanze bei Unwettern im Sommer angeht.

\section{Starker Rückschnitt}

Hierbei werden die Hortensien auf etwa ein Drittel der Gesamtlänge zurückgeschnitten. Als Folge dieses starken Schnittes treibt die Pflanze enorm stark aus und kann dann bei Unwettern auf Grund mangelnder Stabilität auseinanderfallen. Sie benötigt dann unter Umständen Hilfe durch Anbinden oder als Stütze große Staudenhalter. Letztere sind aber nachträglich eingesetzt nicht besonders schön. Hier wäre es sinnvoll, diese Halterung im Frühjahr nach dem Schnitt zu installieren und dann mit der wachsenden Pflanze am Stab nach oben zu ziehen.

\section{Verlängerung der Blütezeit durch einen Sommerschnitt}

Bei Hydrangea paniculata besteht die Möglichkeit, den Strauch zusätzlich im Sommer zu schneiden. In diesem Fall wurde eine als Stamm erzogene Hydrangea paniculata 'Limelight' nach dem Rückschnitt im Frühjahr noch einmal Ende Mai 2020

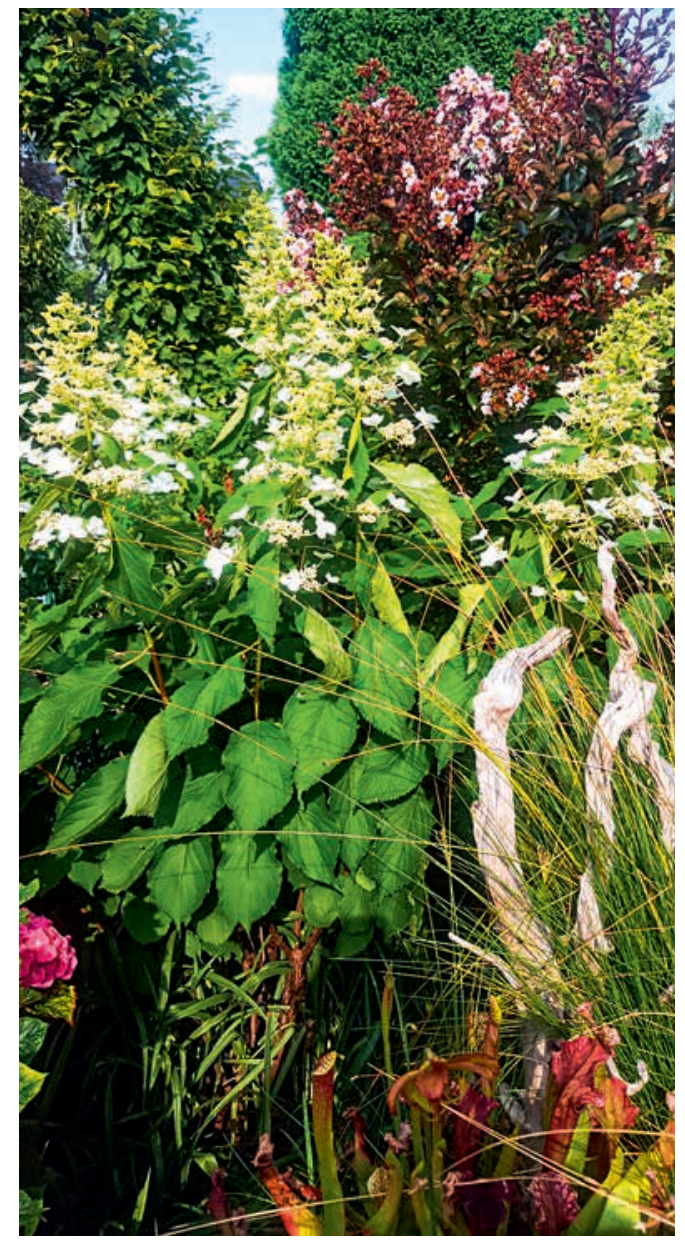

Abb. 2: Herbstblüte nach Sommerschnitt einer Hydrangea paniculata 'Limelight'. (Foto: D. Heukemes)

um etwa ein Drittel an den Neutrieben zurückgeschnitten. Mit Unterstützung von geeignetem Dünger konnte die Pflanze bereits zwei Wochen später wieder einen Neutrieb entwickeln.

Der Sommerschnitt funktioniert nicht nur bei Hydrangea paniculata, sondern auch bei der Wald-Hortensie (Hydrangea arborescens) und deren Sorten. Der Schnitt bewirkt, dass sich die Pflanzen während der Wachstumsphase stabiler aufbauen und der Blütezeitpunkt deutlich nach hinten im Jahr verschoben wird.

Die Aufnahme des Hortensien-Stammes in Abb. 2 wurde am 1. Oktober 2020 gemacht. Den 


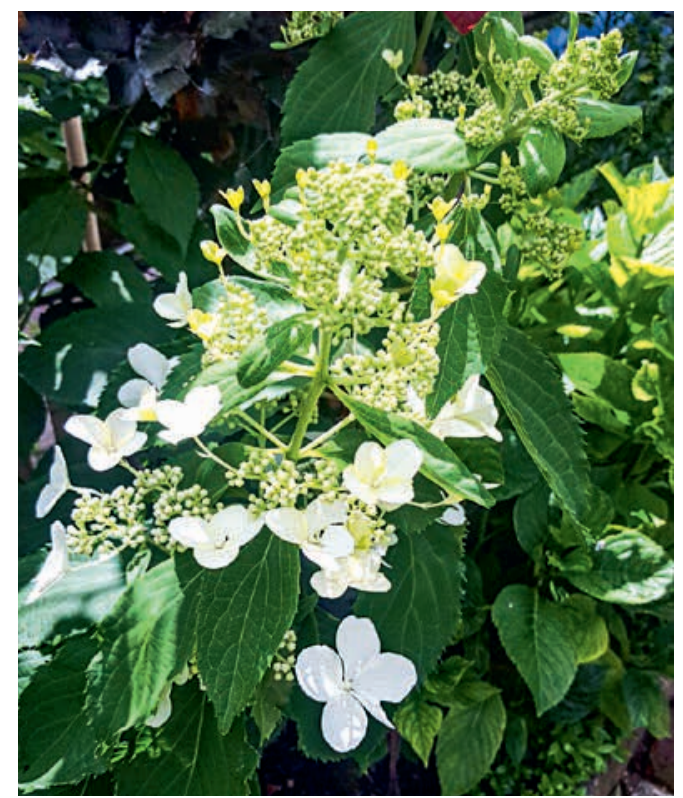

Abb. 3: Hydrangea paniculata 'Levana', die kleinen fertilen Blüten sind hier noch knospig. Im geöffneten Zustand locken sie reichlich Insekten an. (Foto: D. Heukemes)

hier abgebildeten Entwicklungszustand der Blüten hätte die Pflanze bei normalem Schnitt etwa Mitte/Ende Juni. Durch den Sommerschnitt und wegen des damit verbundenen späteren Blütenzeitraums ist die Blüte auch haltbarer. Dies bedeutet, dass die Blütenbälle nicht so schnell ergrünen und länger farbig sind.

\section{Insektenfreundliche Sorten}

Gerade im Zeitalter des Insektensterbens sollte aber auch bei der Verwendung von Rispen-Hortensien im Garten über „nützliche "Sorten nachgedacht werden. Viele alte Sorten wie z.B. 'Limelight' haben fast nur sterile Blüten, die sicherlich für den Menschen sehr schön aussehen, aber für die Insektenwelt keinen Nutzen haben, da sie den Tieren kein Futter bieten. Als Futterpflanzen für Insekten sind dagegen Hydrangea paniculata 'Levana', 'Great Star' und 'Vanille Fraise' geeignet, die neben großen Schaublüten jede Menge fertile Blüten zeigen. Diese Sorten sind für Insekten sehr attraktiv, denn neben Honigbienen und Hummeln findet sich eine große Zahl winziger Wildbienen ein, um sich vom süßen Nektar der Pflanze zu ernähren.

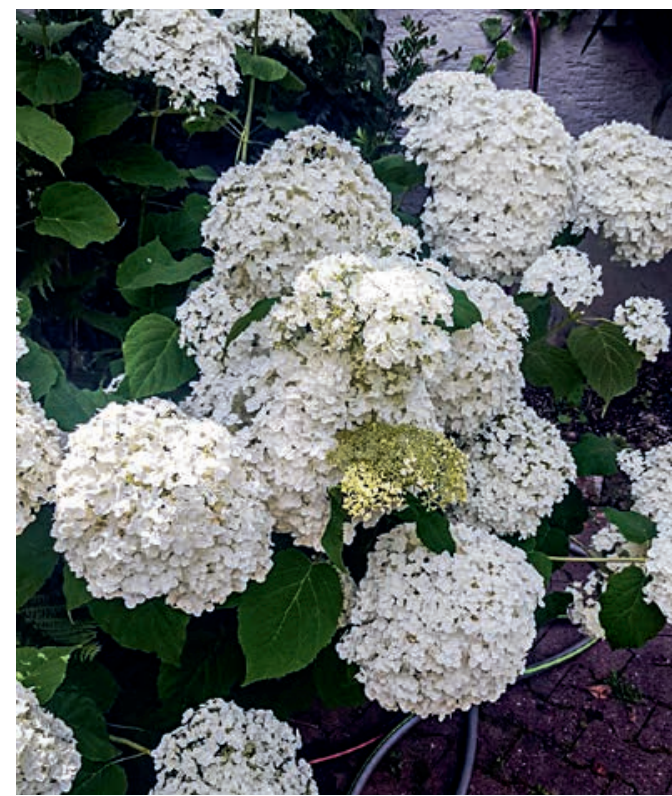

Abb. 4: Sehr attraktiv blüht Hydrangea arborescens ' Strong Annabelle'. (Foto: D. Heukemes)

Bei Hydrangea paniculata 'Levana' wurde auch im vergangenen Jahr 2020 bewusst ein Sommerschnitt vorgenommen, damit zu einem späteren Zeitpunkt im Jahr noch Nektarquellen im Garten zu finden sind.

Mit etwas Achtsamkeit bei der Sortenauswahl in Kombination mit einem eventuellen Sommerschnitt lässt sich also die Hortensien-Blütensaison im Garten verlängern und auch Gutes für unsere heimische Insektenwelt tun.

Derzeit sind im Handel in gut sortierten Gartencentern mit Sicherheit mindestens um die 20 Sorten zu bekommen. Es lohnt sich, nach geeigneten Sorten zu fragen, denn gerade die Kombination aus Insektenfreundlichkeit und Blütenschönheit aus Sicht des Menschen macht den Lebensraum Garten äußerst spannend.

\section{Anschrift des Autors}

Dominik Heunemes, Palmengarten Frankfurt, Siesmayerstr. 61, 60323 Frankfurt,

E-Mail: dominik.heukemes@stadt-frankfurt.de 